\title{
The Complexity of Poor Man's Logic*
}

\author{
Edith Hemaspaandra ${ }^{\dagger}$ \\ Department of Computer Science \\ Rochester Institute of Technology \\ 102 Lomb Memorial Drive \\ Rochester, NY 14623-5608
}

\begin{abstract}
Motivated by description logics, we investigate what happens to the complexity of modal satisfiability problems if we only allow formulas built from literals, $\wedge, \diamond$, and $\square$. Previously, the only known result was that the complexity of the satisfiability problem for $\mathrm{K}$ dropped from PSPACE-complete to coNP-complete (Schmidt-Schauss and Smolka [9] and Donini et al. [3] ). In this paper we show that not all modal logics behave like K. In particular, we show that the complexity of the satisfiability problem with respect to frames in which each world has at least one successor drops from PSPACE-complete to P, but that in contrast the satisfiability problem with respect to the class of frames in which each world has at most two successors remains PSPACE-complete. As a corollary of the latter result, we also solve the open problem from Donini et al.'s complexity classification of description logics 2]. In the last section, we classify the complexity of the satisfiability problem for $\mathrm{K}$ for all other restrictions on the set of operators.
\end{abstract}

\section{Introduction}

Since consistent normal modal logics contain propositional logic, the satisfiability problems for all these logics are automatically NP-hard. In fact, as shown by Ladner [7, many of them are even PSPACE-hard.

But we don't always need all of propositional logic. For example, in some applications we may use only a finite set of propositional variables. Propositional satisfiability thus restricted is in $\mathrm{P}$, and, as shown by Halpern [5], the complexity of satisfiability problems for some modal logics restricted in the same way also decreases. For example, the complexity of S5 satisfiability drops from NP-complete to P. On the other hand, K satisfiability remains PSPACE-complete. The same restriction for linear temporal logics was studied in Demri and Schnoebelen [1].

\footnotetext{
${ }^{*}$ Corrected version of [6]. The changes are in Section [6] where Theorem 6.3 (2) was added to handle two missing cases.

${ }^{\dagger}$ Supported in part by grants NSF-INT-9815095 and NSF-CCR-0311021. Work done in part while visiting the University of Amsterdam. Email: eh@cs.rit.edu.
} 
Restricting the number of propositional variables is not the only propositional restriction on modal logics that occurs in the literature. For example, the description logic $\mathcal{A} \mathcal{L} \mathcal{E}$ can be viewed as multi-modal $\mathrm{K}$ where the formulas are built from literals, $\wedge, \diamond \mathrm{s}$, and $\square \mathrm{s}$.

As in the case of a fixed number of propositional variables, satisfiability for propositional logic for formulas built from literals and $\wedge$ is easily seen to be in P. After all, in that case every propositional formula is the conjunction of literals. Such a formula is satisfiable if and only if there is no propositional variable $p$ such that both $p$ and $\bar{p}$ are conjuncts of the formula.

Hence, satisfiability for modal logics for formulas built from literals, $\wedge, \square$, and $\diamond$ is not automatically NP-hard. Of course, it does not necessarily follow that the complexity of modal satisfiability problems will drop significantly. The only result that was previously known is that the complexity of K satisfiability (i.e., satisfiability with respect to the class of all frames) drops from PSPACEcomplete to coNP-complete. The upper bound was shown by Schmidt-Schauss and Smolka 9, and the lower bound by Donini et al. [3]. It should be noted that these results were shown in the context of description logics (a.k.a. concept languages), so that the notation in these papers is quite different from ours. ${ }^{1}$ In addition, their language contains the constants true and false. However, it is easy to simulate these constants by propositional variables. See Theorem 6.3(1) for details.

In this paper we investigate if it is always the case that the complexity of the satisfiability problem decreases if we only look at formulas that are built from literals, $\wedge, \diamond$, and $\square$, and if so, if there are upper or lower bounds on the amount that the complexity drops.

We will show that not all logics behave like K. Far from it, by looking at simple restrictions on the number of successors that are allowed for each world in a frame, we obtain different levels of complexity, making apparent a subtle interplay between frames and operators. In particular, we will show that

1. The complexity of the satisfiability problem with respect to linear frames drops from NPcomplete to $\mathrm{P}$.

2. The complexity of the satisfiability problem with respect to

3. The complexity of the satisfiability problem with respect to frames in which every world has at least one successor drops from PSPACE-complete to P.

4. The complexity of the satisfiability problem with respect to frames in which every world has at most two successors remains PSPACE-complete.

As a corollary of the last result, we also solve the open problem from Donini et al.'s complexity classification of description logics [2].

In the last section, we completely classify the complexity of the satisfiability problem (with respect to the class of all frames) for all possible restrictions on the set of operators allowed, to

\footnotetext{
${ }^{1}$ Certain description logics can be viewed as syntactic variations of modal logics in the following way: the universal concept corresponds to true, the empty concept corresponds to false, atomic concepts correspond to propositional variables, atomic negation corresponds to propositional negation, complementation corresponds to negation, intersection corresponds to conjunction, union corresponds to disjunction, universal role quantifications correspond to $\square$ operators, and existential role quantifications correspond to $\diamond$ operators 8 ]
} 
gain more insight in the sources of complexity for modal logics. It turns out that the restriction studied in this paper, which we will call poor man's logic, is the only (constant-free) fragment whose satisfiability problem is so unusual. For all other restrictions, the satisfiability problems are PSPACE-complete, NP-complete, or in P. These are exactly the complexity classes that one would expect to show up in this context.

\section{Definitions}

We will first briefly review syntax, Kripke semantics, and some basic terminology for modal logic.

\section{Syntax}

The set of $\mathcal{L}$ formulas is inductively defined as follows. (As usual, we assume that we have a countably infinite set of propositional variables.)

- $p$ and $\bar{p}$ are $\mathcal{L}$ formulas for every propositional variable $p$,

- if $\phi$ and $\psi$ are $\mathcal{L}$ formulas, then so are $\phi \wedge \psi$ and $\phi \vee \psi$, and

- if $\phi$ is an $\mathcal{L}$ formula, then $\square \phi$ and $\diamond \phi$ are $\mathcal{L}$ formulas.

We will identify $\overline{\bar{p}}$ with $p$.

The modal depth of a formula $\phi$ (denoted by $m d(\phi))$ is the depth of nesting of the modal operators $\square$ and $\diamond$.

\section{Semantics}

A frame is a tuple $F=<W, R>$ where $W$ is a non-empty set of possible worlds, and $R$ is a binary relation on $W$ called the accessibility relation.

A model is of the form $M=\langle W, R, \pi>$ such that $\langle W, R>$ is a frame (we say that $M$ is based on this frame), and $\pi$ is a function from the set of propositional variables to $\operatorname{Pow}(W)$ : a valuation, i.e., $\pi(p)$ is the set of worlds in which $p$ is true. For $\phi$ an $\mathcal{L}$ formula, we will write $M, w \models \phi$ for $\phi$ is true /satisfied at $w$ in $M$. The truth relation $\models$ is defined with induction on $\phi$ in the following way.

- $M, w \models p$ iff $w \in \pi(p)$ for $p$ a propositional variable.

- $M, w \models \bar{p}$ iff $w \notin \pi(p)$ for $p$ a propositional variable.

- $M, w \models \phi \wedge \psi$ iff $M, w \models \phi$ and $M, w \models \psi$.

- $M, w \models \phi \vee \psi$ iff $M, w \models \phi$ or $M, w \models \psi$.

- $M, w \models \square \phi$ iff $\forall w^{\prime} \in W\left[w R w^{\prime} \Rightarrow M, w^{\prime} \models \phi\right]$.

- $M, w \models \diamond \phi$ iff $\exists w^{\prime} \in W\left[w R w^{\prime}\right.$ and $\left.M, w^{\prime} \models \phi\right]$. 
The size of a model or frame is the number of worlds in the model or frame.

The notion of satisfiability can be extended to models, frames, and classes of frames in the following way. $\phi$ is satisfied in model $M$ if $M, w \models \phi$ for some world $w$ in $M, \phi$ is satisfiable in frame $F$ ( $F$ satisfiable) if $\phi$ is satisfied in $M$ for some model $M$ based on $F$, and $\phi$ is satisfiable with respect to class of frames $\mathcal{F}(\mathcal{F}$ satisfiable $)$ if $\phi$ is satisfiable in some frame $F \in \mathcal{F}$.

As usual, we will look at satisfiability with respect to classes of frames. For a class of frames $\mathcal{F}$, the satisfiability problem with respect to $\mathcal{F}$ is the problem of determining, given an $\mathcal{L}$ formula $\phi$, whether $\phi$ is $\mathcal{F}$ satisfiable. For a complete logic $L$, we will sometimes view $L$ as the class of frames where $L$ is valid. For example, we will speak of $\mathrm{K}$ satisfiability when we mean satisfiability with respect to all frames. Likewise, we will on occasion identify a class of frames with its logic, i.e., with the set of formulas valid on this class of frames.

\section{Poor Man's Logic}

The set of poor man's formulas is the set of $\mathcal{L}$ formulas that do not contain $\vee$. The poor man's satisfiability problem with respect to $\mathcal{F}$ is the problem of determining, given a poor man's formula $\phi$, whether $\phi$ is $\mathcal{F}$ satisfiable.

In poor man's language, we will view $\wedge$ as a multi-arity operator, and we will assume that all conjunctions are "flattened," that is, a conjunct will not be a conjunction. Thus, a formula $\phi$ in this language is of the following form: $\phi=\square \psi_{1} \wedge \cdots \wedge \square \psi_{k} \wedge \diamond \xi_{1} \wedge \cdots \wedge \diamond \xi_{m} \wedge \ell_{1} \wedge \cdots \wedge \ell_{s}$, where the $\ell_{i}$ s are literals.

In all but the last section of this paper, we will compare the complexity of satisfiability to the complexity of poor man's satisfiability with respect to the same class of frames. We are interested in simple restrictions on the number of successor worlds that are allowed. Let $\mathcal{F}_{\leq 1}, \mathcal{F}_{\leq 2}$, and $\mathcal{F}_{\geq 1}$ be the classes of frames in which every world has at most one, at most two, and at least one successor, respectively. (Note that $\mathcal{F}_{\geq 1}$ corresponds to the logic KD.)

\section{Poor Man's Versions of NP-complete Satisfiability Problems}

We already know that the poor man's version of an NP-complete modal satisfiability problem can be in P. Look for example at satisfiability with respect to the class of frames where no world has a successor. This is plain propositional logic in disguise, and it inherits the complexity behavior of propositional logic. As mentioned in the introduction, the complexity of satisfiability drops from NP-complete to P.

In this section, we will give an example of a non-trivial modal logic with the same behavior. We will show that the poor man's version of satisfiability with respect to linear frames is in P. In contrast, we will also give a very simple example of a modal logic where the complexity of poor man's satisfiability remains NP-complete.

Theorem 3.1 Satisfiability with respect to $\mathcal{F}_{\leq 1}$ is NP-complete and poor man's satisfiability with respect to $\mathcal{F}_{\leq 1}$ is in $P$. 
Proof. Clearly, $\mathcal{F}_{\leq 1}$ satisfiability is in NP (and thus NP-complete), since every satisfiable formula is satisfiable on a linear frame with $\leq m d(\phi)$ worlds, where $m d(\phi)$ is the modal depth of $\phi$. This immediately gives the following NP algorithm for $\mathcal{F}_{\leq 1}$ satisfiability: Guess a linear frame of size $\leq m d(\phi)$, and for every world in the frame, guess a valuation on the propositional variables that occur in $\phi$. Accept if and only if the guessed model satisfies $\phi$.

It is easy to see that the following polynomial-time algorithm decides poor man's satisfiability with respect to $\mathcal{F}_{\leq 1}$. Let $\phi=\square \psi_{1} \wedge \cdots \wedge \square \psi_{k} \wedge \diamond \xi_{1} \wedge \cdots \wedge \diamond \xi_{m} \wedge \ell_{1} \wedge \cdots \wedge \ell_{s}$, where the $\ell_{i}$ s are literals. $\phi$ is $\mathcal{F}_{\leq 1}$ satisfiable if and only if

- $\ell_{1} \wedge \cdots \wedge \ell_{s}$ is satisfiable (that is, for all $i$ and $j, \ell_{i} \neq \overline{\ell_{j}}$ ), and

- $\quad-m=0$, (that is, $\phi$ does not contain conjuncts of the form $\diamond \xi$, in which case the formula is satisfied in a world with no successors), or

$-\bigwedge_{i=1}^{k} \psi_{i} \wedge \bigwedge_{i=1}^{m} \xi_{i}$ is $\mathcal{F}_{\leq 1}$ satisfiable (the world has exactly one successor).

From the previous example, you might think that the poor man's versions of logics with the polysize frame property are in P, or even that the poor man's versions of all NP-complete satisfiability problems are in P. Not so. The following theorem gives a very simple counterexample.

Theorem 3.2 Satisfiability and poor man's satisfiability with respect to the frame.$\bullet^{+} \cdot$ are NPcomplete.

Proof. Because the frame is finite, both satisfiability problems are in NP. Thus it suffices to show that poor man's satisfiability with respect to.

Since we are working with a fragment of propositional modal logic, it is extremely tempting to try to reduce from an NP-complete propositional satisfiability problem. However, because poor man's logics contain only a fragment of propositional logic, these logics don't behave like propositional logic at all. Because of this, propositional satisfiability problems are not the best choice of problems to reduce from. In fact, they are particularly confusing.

It turns out that it is much easier to reduce a partitioning problem to our poor man's satisfiability problem. We will reduce from the following well-known NP-complete problem.

GRAPH 3-COLORABILITY: Given an undirected graph $G$, can you color every vertex of the graph using only three colors in such a way that vertices connected by an edge have different colors?

Suppose $G=(V, E)$ where $V=\{1,2, \ldots, n\}$. We introduce a propositional variable $p_{e}$ for every edge $e$. The three leaves of 0 will correspond to the three colors. To ensure that adjacent vertices in the graph end up in different leaves, we will make sure that the smaller endpoint of $e$ satisfies $p_{e}$ and that the larger endpoint of $e$ satisfies $\overline{p_{e}}$.

The requirements for vertex $i$ are given by the following formula:

$$
\psi_{i}=\bigwedge\left\{p_{e} \mid e=\{i, j\} \text { and } i<j\right\} \wedge \bigwedge\left\{\overline{p_{e}} \mid e=\{i, j\} \text { and } i>j\right\} .
$$


Define $f(G)=\bigwedge_{i=1}^{n} \diamond \psi_{i}$.

$f$ is clearly computable in polynomial time. To show that $f$ is indeed a reduction from GRAPH

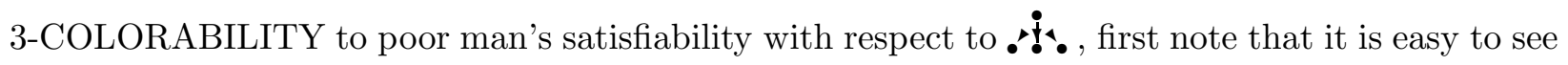
that for every set $V^{\prime} \subseteq V$, the following holds: $\bigwedge_{i \in V^{\prime}} \psi_{i}$ is satisfiable if and only if no two vertices in $V^{\prime}$ are connected by an edge.

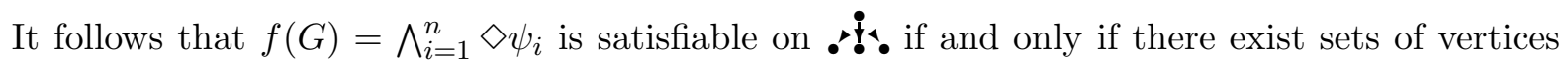
$V_{1}, V_{2}$, and $V_{3}$ such that $V=V_{1} \cup V_{2} \cup V_{3}$ and $\bigwedge_{i \in V_{j}} \psi_{i}$ is satisfiable for $j \in\{1,2,3\}$. This holds if and only if there exist sets of vertices $V_{1}, V_{2}$, and $V_{3}$ such that $V=V_{1} \cup V_{2} \cup V_{3}$ and no two vertices in $V_{j}$ are adjacent for $j \in\{1,2,3\}$, which is the case if and only if $G$ is 3-colorable. (We obtain a coloring by coloring each vertex $v$ by the smallest $j$ such that $v \in V_{j}$.)

\section{Poor Man's Versions of PSPACE-complete Satisfiability Prob- lems}

It is well-known that the satisfiability problems for many modal logics including $\mathrm{K}$ are PSPACEcomplete [7. We also know that poor man's satisfiability for $\mathrm{K}$ is coNP-complete [9, 3]. That is, in that particular case the complexity of the satisfiability problem drops from PSPACE-complete to coNP-complete. Is this the general pattern? We will show that this is not the case. We will give an example of a logic where the complexity of the satisfiability problem drops from PSPACE-complete all the way down to $\mathrm{P}$, and another example in which the complexity of both the satisfiability and the poor man's satisfiability problems are PSPACE-complete. Both examples are really close to K; they are satisfiability with respect to $\mathcal{F}_{\geq 1}$ and $\mathcal{F}_{\leq 2}$, respectively.

We will first consider $\mathcal{F}_{\geq 1}$. This logic is very close to $K$ and it should come as no surprise that the complexity of $\mathcal{F}_{\geq 1}$ satisfiability and $\mathrm{K}$ satisfiability are the same. It may come as a surprise to learn that poor man's satisfiability with respect to $\mathcal{F}_{\geq 1}$ is in P. It is easy to show that poor man's satisfiability with respect to $\mathcal{F}_{\geq 1}$ is in coNP, because the following function $f$ reduces the poor man's satisfiability problem with respect to $\mathcal{F}_{\geq 1}$ to the poor man's satisfiability problem for K.

$$
f(\phi)=\phi \wedge \bigwedge_{i=0}^{m d(\phi)} \square^{i} \diamond q,
$$

where $q$ is a propositional variable not in $\phi$. The formula ensures that every world in the relevant part of the $\mathrm{K}$ frame has at least one successor.

It is very surprising that poor man's satisfiability with respect to $\mathcal{F}_{\geq 1}$ is in $\mathrm{P}$, because the relevant part of the $\mathcal{F}_{\geq 1}$ frame may require an exponential number of worlds to satisfy a formula in poor man's language. For example, consider the following formula:

$$
\diamond \square \square p_{1} \wedge \diamond \square \square \overline{p_{1}} \wedge \square\left(\diamond \square p_{2} \wedge \diamond \square \overline{p_{2}}\right) \wedge \square \square\left(\diamond p_{3} \wedge \diamond \overline{p_{3}}\right)
$$

If this formula is satisfiable in world $w$, then for every assignment to $p_{1}, p_{2}$, and $p_{3}$, there exists a world reachable in three steps from $w$ that satisfies that assignment. 
In its general version, the formula becomes

$$
\phi \exp =\bigwedge_{i=1}^{n} \square^{i-1}\left(\diamond \square^{n-i} p_{i} \wedge \diamond \square^{n-i} \overline{p_{i}}\right) .
$$

The formula is of length polynomial in $n$ and forces the relevant part of the model to be of exponential size.

Now that we have seen how surprising it is that poor man's satisfiability with respect to $\mathcal{F}_{\geq 1}$ is in $\mathrm{P}$, let's prove it.

Theorem 4.1 Satisfiability with respect to $\mathcal{F}_{\geq 1}$ is PSPACE-complete and poor man's satisfiability with respect to $\mathcal{F}_{\geq 1}$ is in $P$.

Proof. The proof that satisfiability with respect to $\mathcal{F}_{\geq 1}$ is PSPACE-complete is very close to the proof that K satisfiability is PSPACE-complete [7] and therefore omitted.

For the poor man's satisfiability problem, note that a simplified version of Ladner's PSPACE upper bound construction for $\mathrm{K}$ can be used to show the following.

Let $\phi=\square \psi_{1} \wedge \cdots \wedge \square \psi_{k} \wedge \diamond \xi_{1} \wedge \cdots \wedge \diamond \xi_{m} \wedge \ell_{1} \wedge \cdots \wedge \ell_{s}$, where the $\ell_{i}$ s are literals. $\phi$ is $\mathcal{F}_{\geq 1}$ satisfiable if and only if

1. $\ell_{1} \wedge \cdots \wedge \ell_{s}$ is satisfiable,

2. for all $j, \psi_{1} \wedge \cdots \wedge \psi_{k} \wedge \xi_{j}$ is $\mathcal{F}_{\geq 1}$ satisfiable, and

3. $\psi_{1} \wedge \cdots \wedge \psi_{k}$ is $\mathcal{F}_{\geq 1}$ satisfiable. (only relevant when $m=0$.)

Note that this algorithm takes exponential time and polynomial space. Of course, we already know that poor man's satisfiability with respect to $\mathcal{F}_{\geq 1}$ is in PSPACE, since satisfiability with respect to $\mathcal{F}_{\geq 1}$ is in PSPACE. How can this PSPACE algorithm help to prove that poor man's satisfiability with respect to $\mathcal{F}_{\geq 1}$ is in $\mathrm{P}$ ?

Something really surprising happens here. We will prove that for every poor man's formula $\phi, \phi$ is $\mathcal{F}_{\geq 1}$ satisfiable if and only if (the conjunction of) every pair of (not necessary different) conjuncts of $\phi$ is $\mathcal{F}_{\geq 1}$ satisfiable. Using dynamic programming, we can compute all pairs of subformulas of $\phi$ that are $\mathcal{F}_{\geq 1}$ satisfiable in polynomial time. This proves the theorem. It remains to show that for every poor man's formula $\phi, \phi$ is $\mathcal{F}_{\geq 1}$ satisfiable if and only if every pair of conjuncts of $\phi$ is $\mathcal{F}_{\geq 1}$ satisfiable. We will prove this claim by induction on $\operatorname{md}(\phi)$, the modal depth of $\phi$. In the proof, we will write "satisfiable" for "satisfiable with respect to $\mathcal{F}_{\geq 1}$."

If $\operatorname{md}(\phi)=0, \phi$ is a conjunction of literals. In that case $\phi$ is not satisfiable if and only if there exist $i$ and $j$ such that $\ell_{i}=\overline{\ell_{j}}$. This immediately implies our claim.

For the induction step, suppose $\phi=\square \psi_{1} \wedge \cdots \wedge \square \psi_{k} \wedge \diamond \xi_{1} \wedge \cdots \wedge \diamond \xi_{m} \wedge \ell_{1} \wedge \cdots \wedge \ell_{s}$ (where the $\ell_{i}$ s are literals), $m d(\phi) \geq 1$, and suppose that our claim holds for all formulas of modal depth $<m d(\phi)$. Suppose for a contradiction that $\phi$ is not satisfiable, though every pair of conjuncts of $\phi$ is satisfiable. Then, by the Ladner-like construction given above, we are in one of the following three cases: 
1. $\ell_{1} \wedge \cdots \wedge \ell_{s}$ is not satisfiable,

2. for some $j, \psi_{1} \wedge \cdots \wedge \psi_{k} \wedge \xi_{j}$ is not satisfiable, or

3. $\psi_{1} \wedge \cdots \wedge \psi_{k}$ is not satisfiable.

By induction, it follows immediately that we are in one of the following four cases:

1. There exist $i, i^{\prime}$ such that $\ell_{i} \wedge \ell_{i^{\prime}}$ is not satisfiable,

2. there exist $i, i^{\prime}$ such that $\psi_{i} \wedge \psi_{i^{\prime}}$ is not satisfiable,

3. there exist $i, j$ such that $\psi_{i} \wedge \xi_{j}$ is not satisfiable, or

4. there exists a $j$ such that $\xi_{j} \wedge \xi_{j}$ is not satisfiable.

If we are in case $2, \square \psi_{i} \wedge \square \psi_{i^{\prime}}$ is not satisfiable. In case $3, \square \psi_{i} \wedge \diamond \xi_{j}$ is not satisfiable. In case $4, \diamond \xi_{j} \wedge \diamond \xi_{j}$ is not satisfiable. So in each case we have found a pair of conjuncts of $\phi$ that is not satisfiable, which contradicts the assumption.

Why doesn't the same construction work for K? It is easy enough to come up with a counterexample. For example, $\{\square p, \square \bar{p}, \diamond q\}$ is not satisfiable, even though every pair is satisfiable. The deeper reason is that we have some freedom in $K$ that we don't have in $\mathcal{F}_{\geq 1}$. Namely, on a $K$ frame a world can have successors or no successors. This little bit of extra freedom is enough to encode coNP in poor man's language.

Theorem 3.2 showed that poor man's satisfiability can be as hard as satisfiability for NPcomplete logics. In light of the fact that poor man's satisfiability for $\mathrm{K}$ is coNP-complete and poor man's satisfiability with respect to $\mathcal{F}_{\geq 1}$ is even in $\mathrm{P}$, you might wonder if the complexity of PSPACE-complete logics always decreases.

To try to keep the complexity as high as possible, it makes sense to look at frames in which each world has a restricted number of successors, as in the construction of Theorem 3.2. Because we want the logic to be PSPACE-complete, we also need to make sure that the frames can simulate binary trees. The obvious class of frames to look at is $\mathcal{F}_{\leq 2}$ - the class of frames in which each world has at most two successors. This gives us the desired example.

Theorem 4.2 Satisfiability and poor man's satisfiability with respect to $\mathcal{F}_{\leq 2}$ are PSPACEcomplete.

Proof. Satisfiability with respect to $\mathcal{F}_{\leq 2}$ is PSPACE-complete by pretty much the same proof as the PSPACE-completeness proof for K [7]. To show that the poor man's version remains PSPACEcomplete, first note that a formula is $\mathcal{F}_{\leq 2}$ satisfiable if and only if it is satisfiable in the root of a binary tree. Stockmeyer [10] showed that the set of true quantified 3CNF formulas is PSPACEcomplete. Using padding, it is immediate that the following variation of this set is also PSPACEcomplete. 
QUANTIFIED 3SAT: Given a quantified Boolean formula $\exists p_{1} \forall p_{2} \exists p_{3} \cdots \exists p_{n-1} \forall p_{n} \phi$, where $\phi$ is a propositional formula over $p_{1}, \ldots, p_{n}$ in $3 \mathrm{CNF}$ (that is, a formula in conjunctive normal form with exactly 3 literals per clause), is the formula true?

We will reduce QUANTIFIED 3SAT to poor man's satisfiability with respect to binary trees. To simulate the quantifiers, we need to go back to the formula that forces models to be of exponential size.

$$
\phi_{\exp }=\bigwedge_{i=1}^{n} \square^{i-1}\left(\diamond \square^{n-i} p_{i} \wedge \diamond \square^{n-i} \overline{p_{i}}\right) .
$$

$\phi_{\text {exp }}$ is clearly satisfiable in the root of a binary tree and if $\phi_{\exp }$ is satisfied in the root of a binary tree, the worlds of depth $\leq n$ form a complete binary tree of depth $n$ and every assignment to $p_{1}, \ldots, p_{n}$ occurs exactly once in a world at depth $n$. We will call the worlds at depth $n$ the assignment-worlds.

The assignment-worlds in a subtree rooted at a world at distance $i \leq n$ from the root are constant with respect to the value of $p_{i}$. It follows that $\exists p_{1} \forall p_{2} \exists p_{3} \cdots \exists p_{n-1} \forall p_{n} \phi \in$ QUANTIFIED 3SAT if and only if $\phi \exp \wedge(\diamond \square)^{n / 2} \phi$ is satisfiable with respect to binary trees.

This proves that satisfiability for $\mathcal{F}_{\leq 2}$ is PSPACE-hard, but it does not prove that the poor man's version is PSPACE-hard. Recall that $\phi$ is in 3CNF and thus not a poor man's formula.

Below, we will show how to label all assignment-worlds where $\phi$ does not hold by $f$ (for false). It then suffices to add the conjunct $(\diamond \square)^{n / 2} \bar{f}$ to obtain a reduction.

How can we label all assignment-worlds where $\phi$ does not hold by $f$ ? Let $k$ be such that $\phi=\psi_{1} \wedge \psi_{2} \wedge \cdots \wedge \psi_{k}$, where each $\psi_{i}$ is the disjunction of exactly 3 literals: $\psi_{i}=\ell_{i 1} \vee \ell_{i 2} \vee \ell_{i 3}$. We assume without loss of generality that $n$ is even and that each $\psi_{i}$ contains 3 different propositional variables.

For every $i$, we will label all assignment-worlds where $\psi_{i}$ does not hold by $f$. Since $\psi_{i}=$ $\ell_{i 1} \vee \ell_{i 2} \vee \ell_{i 3}$, this implies that we have to label all assignment-worlds where $\overline{\ell_{i 1}} \wedge \overline{\ell_{i 2}} \wedge \overline{\ell_{i 3}}$ holds by $f$. In general, this cannot be done in poor man's logic, but in this special case we are able to do it, because the relevant part of the model is completely fixed by $\phi$ exp.

As a warm-up, first consider how you would label all assignment-worlds where $\overline{p_{3}}$ holds by $f$. This is easy; add the conjunct

$$
\square \square \diamond \square^{n-3}\left(\overline{p_{3}} \wedge f\right) .
$$

You can label all assignment-worlds where $\overline{p_{3}} \wedge p_{5}$ holds as follows:

$$
\square \square \diamond \square \diamond \square^{n-5}\left(\overline{p_{3}} \wedge p_{5} \wedge f\right) .
$$

This can easily be generalized to a labeling for $\overline{p_{3}} \wedge p_{5} \wedge \overline{p_{8}}$ :

$$
\square \square \diamond \square \diamond \square \square \diamond \square^{n-8}\left(\overline{p_{3}} \wedge p_{5} \wedge \overline{p_{8}} \wedge f\right) .
$$

Note that we can write the previous formula in the following suggestive way:

$$
\square^{3-1} \diamond \square^{5-3-1} \diamond \square^{8-5-1} \diamond \square^{n-8}\left(\overline{p_{3}} \wedge p_{5} \wedge \overline{p_{8}} \wedge f\right) .
$$


In general, suppose you want to label all assignment-worlds where $\ell_{1} \wedge \ell_{2} \wedge \ell_{3}$ hold by $f$, where $\ell_{1}, \ell_{2}$, and $\ell_{3}$ are literals. Suppose that $\ell_{1}, \ell_{2}$, and $\ell_{3}$ 's propositional variables are $p_{a}, p_{b}$, and $p_{c}$, respectively. Also suppose that $a<b<c$. The labeling formula label_false $\left(\ell_{1} \wedge \ell_{2} \wedge \ell_{3}\right)$ is defined as follows.

$$
\text { label_false }\left(\ell_{1} \wedge \ell_{2} \wedge \ell_{3}\right)=\square^{a-1} \diamond \square^{b-a-1} \diamond \square^{c-b-1} \diamond \square^{n-c}\left(\ell_{1} \wedge \ell_{2} \wedge \ell_{3} \wedge f\right) .
$$

If label_false $\left(\ell_{1} \wedge \ell_{2} \wedge \ell_{3}\right)$ is satisfied in the root of a complete binary tree, then there exist at least $2^{n-3}$ worlds at depth $n$ such that $\left(\ell_{1} \wedge \ell_{2} \wedge \ell_{3} \wedge f\right)$ holds.

If $\phi_{\text {exp }}$ is satisfied in the root of a binary tree, then the worlds of depth $\leq n$ form a complete binary tree and there are exactly $2^{n-3}$ assignment-worlds such that $\left(\ell_{1} \wedge \ell_{2} \wedge \ell_{3}\right)$ holds.

It follows that if $\phi_{\exp }$ is satisfied in the root of a binary tree, then label_false $\left(\ell_{1} \wedge \ell_{2} \wedge \ell_{3}\right)$ is satisfied in the root if and only if $f$ holds in every assignment-world where $\left(\ell_{1} \wedge \ell_{2} \wedge \ell_{3}\right)$ holds.

Thus, the following function $g$ is a reduction from QUANTIFIED 3SAT to poor man's satisfiability with respect to $\mathcal{F}_{\leq 2}$.

$$
g\left(\exists p_{1} \forall p_{2} \exists p_{3} \cdots \exists p_{n-1} \forall p_{n} \phi\right)=\phi_{\exp } \wedge \bigwedge_{i=1}^{k} \text { label_false }\left(\overline{\ell_{i 1}} \wedge \overline{\ell_{i 2}} \wedge \overline{\ell_{i 3}}\right) \wedge(\diamond \square)^{n / 2} \bar{f} .
$$

Why doesn't the construction of Theorem 4.2 work for K? A formula that is satisfiable in a world with exactly two successors is also satisfiable in a world with more than two successors. Because of this, the label_false formula will not necessarily label all assignment-worlds where $\phi$ does not hold by $f$. For a very simple example, consider the formula

$$
\diamond p \wedge \diamond \bar{p} \wedge \diamond(p \wedge f) \wedge \diamond(\bar{p} \wedge f) \wedge \diamond \bar{f}
$$

This formula is not $\mathcal{F}_{\leq 2}$ satisfiable, since both the $p$ successor and the $\bar{p}$ successor are labeled $f$. However, this formula is satisfiable in a world with three successors, satisfying $p \wedge f, \bar{p} \wedge f$, and $\bar{f}$, respectively.

\section{$5 \quad \mathcal{A L E \mathcal { N }}$ Satisfiability is PSPACE-complete}

In the introduction, we mentioned that poor man's logic is closely related to certain description logics. Donini et al. 2] almost completely characterize the complexity of the most common description logics. The only language they couldn't completely characterize is $\mathcal{A} \mathcal{L} \mathcal{E}$. $\mathcal{A} \mathcal{L} \mathcal{E}$ is $\mathcal{A} \mathcal{L}$ (the poor man's version of multi-modal K) with number restrictions. Number restrictions are of the form $(\leq n)$ and $(\geq n)$. $(\leq n)$ is true if and only if a world has $\leq n$ successors and $(\geq n)$ is true if and only if a world has $\geq n$ successors.

In 2], it was shown that $\mathcal{A} \mathcal{L} \mathcal{E N}$ satisfiability is in PSPACE, assuming that the number restrictions are given in unary. Tobies [1] showed that $\mathcal{A L E N}$ satisfiability remains in PSPACE if the number restrictions are given in binary. The best lower bound for $\mathcal{A L E \mathcal { N }}$ satisfiability was the coNP lower bound that is immediate from the fact that this is an extension of $\mathcal{A L E}$.

We will use Theorem 4.2 to prove PSPACE-hardness for a very restricted version of $\mathcal{A L \mathcal { L N }}$. 
Theorem 5.1 Satisfiability for the poor man's version of $K$ extended with the number restriction $(\leq 2)$ is PSPACE-hard.

Proof. The reduction from poor man's satisfiability with respect to $\mathcal{F}_{\leq 2}$ is obvious. It suffices to use the number restriction $(\leq 2)$ to make sure that every world in the relevant part of the model has at most two successors. Let $m d(\phi)$ be the modal depth of $\phi$. All worlds that are of importance to the satisfiability of $\phi$ are at most $m d(\phi)$ steps away from the root. The reduction is as follows:

$$
f(\phi)=\phi \wedge \bigwedge_{i=0}^{m d(\phi)} \square^{i}(\leq 2)
$$

Combining this with the PSPACE upper bound from [2] completely characterizes the complexity of $\mathcal{A L \mathcal { L N }}$ satisfiability.

Corollary $5.2 \mathcal{A L E N}$ satisfiability is PSPACE-complete.

\section{Other Restrictions on the Set of Operators}

As mentioned in the introduction, restricting the modal language in the way that we have, i.e., looking at formulas built from literals, $\wedge, \square$, and $\diamond$, was motivated by the fact that this restriction occurs in description logics and also by the rather bizarre complexity behavior of this fragment.

From a more technical point of view however, we might well wonder what happens to other restrictions on the set of operators allowed. After all, who is to say which sublanguages will be useful in the future? Also, we might hope to gain more insight in the sources of complexity for modal logics by looking at different sublanguages.

For $S \subseteq\left\{\neg,{ }^{-}, \wedge, \vee, \square, \diamond\right.$, true, false $\}$, let $\mathcal{L}(S)$ denote the modal language whose formulas are built from an infinite set of propositional variables and operators from $S$. We will write ${ }^{-}$for propositional negation, and $\neg$ for general negation. So, our "old" language $\mathcal{L}$ will be denoted by $\mathcal{L}(\{-, \wedge, \vee, \square, \diamond\})$, and poor man's language by $\mathcal{L}(\{-, \wedge, \square, \diamond\})$.

In this section, we will completely characterize the complexity of $\mathcal{L}(S)$ satisfiability (with respect to the class of all frames), for every $S \subseteq\{\neg,-, \wedge, \vee, \square, \diamond$, true, false $\}$.

Since there are $2^{8}$ subsets, this may seem to be a daunting task. But we will see that there are only four possibilities for the complexity of these satisfiability problems: P, NP-complete, coNPcomplete, and PSPACE-complete. Also, there are not many surprises: Languages that contain a complete basis for modal logic obviously have PSPACE-complete satisfiability problems (Theorem 6.1), languages that contain a complete basis for propositional logic, but not for modal logic have NP-complete satisfiability problems (Theorem 6.2), and poor man's logic is the only constantfree coNP-complete case (Theorem 6.3(1)). All other cases are in $\mathrm{P}$ (Theorem 6.4 6.8), except for the surprise that $\mathcal{L}(\{\wedge, \square, \diamond$, false $\})$ satisfiability is coNP-complete (Theorem 6.3 (2)) and that $\mathcal{L}(\{\wedge, \vee, \square, \diamond$, false $\})$ satisfiability is PSPACE-complete (Theorem 6.5). 
Theorem 6.1 If $S \subseteq\{\neg,-, \wedge, \vee, \square, \diamond$, true, false $\}$ contains $\{\neg, \wedge, \square\}, \quad\{\neg, \wedge, \diamond\}, \quad\{\neg, \vee, \square\}$, $\{\neg, \vee, \diamond\}$, or $\{-\wedge, \vee, \square, \diamond\}$, then $\mathcal{L}(S)$ satisfiability is PSPACE-complete.

Proof. It is easy to see that these five sets are exactly the minimal complete bases for modal logic. All these satisfiability problems are polynomial-time equivalent, and their PSPACE-completeness follows immediately from Ladner [7].

Theorem 6.2 1. If $S \subseteq\{\neg,-, \wedge, \vee$, true, false $\}$ and $S$ contains $\{\neg, \vee\}$ or $\{\neg, \wedge\}$, then $\mathcal{L}(S)$ satisfiability is NP-complete.

2. If $S$ contains $\left\{{ }^{-}, \wedge, \vee\right\}$ and $S$ is a subset of $\left\{{ }^{-}, \wedge, \vee, \square\right.$, true, false $\}$ or of $\{-\wedge, \vee, \diamond$, true, false $\}$, then $\mathcal{L}(S)$ satisfiability is NP-complete.

Proof. First note that all these cases are clearly NP-hard, because they contain propositional satisfiability. It remains to show that $\mathcal{L}(S)$ satisfiability is in NP if $S=\left\{\neg,{ }^{-}, \wedge, \vee\right.$, true, false $\}$, $\{-, \wedge, \vee, \square$, true, false $\}$, or $\{-, \wedge, \vee, \diamond$, true, false $\}$.

The first case is exactly propositional satisfiability and thus in NP.

For the second case, an $\mathcal{L}(\{-, \wedge, \vee, \square$, true, false $\})$ formula is satisfiable if and only if substituting true for every outermost $\square \psi$ subformula gives a propositionally satisfiable formula.

For the last case, the following NP algorithm decides $\mathcal{L}(\{-, \wedge, \vee, \diamond$, true, false $\})$ satisfiability. Given a formula $\phi$, guess a valuation on all the subformulas of $\phi$ and accept if and only if this valuation makes $\phi$ true, the valuation is propositionally consistent, and for all $\diamond \psi$ that are set to true, $\psi$ is satisfiable. It is crucial that we do not have to verify anything if $\diamond \psi$ is set to false in the valuation, because $\diamond \psi$ can only occur positively.

Theorem 6.3 1. If $\{-, \wedge, \square, \diamond\} \subseteq S \subseteq\{-, \wedge, \square, \diamond$, true, false $\}$, then $\mathcal{L}(S)$ satisfiability is coNPcomplete.

2. If $\{\wedge, \square, \diamond$,false $\} \subseteq S \subseteq\left\{{ }^{-}, \wedge, \square, \diamond\right.$, true, false $\}$, then $\mathcal{L}(S)$ satisfiability is coNP-complete.

Proof. As mentioned in the introduction, the $\mathcal{L}(\{-, \wedge, \square, \diamond$, true, false $\})$ case follows from [9, 3].

It is easy to see that $\mathcal{L}(\{-, \wedge, \square, \diamond$, true, false $\})$ satisfiability with respect to any class of frames $\mathcal{F}$ is polynomial-time reducible to $\mathcal{L}(\{-, \wedge, \square, \diamond\})$ satisfiability with respect to the same class of frames: Introduce two new variables $t$ and $f$ to simulate true and false, respectively, and define reduction $g$ as follows.

$$
g(\phi)=\phi[\text { true }:=t, \text { false }:=f] \wedge \bigwedge_{i=0}^{m d(\phi)} \square^{i}(t \wedge \bar{f}) .
$$

This completes the proof of part (1), i.e., poor man's logic with or without constants.

It remains to show that $\mathcal{L}(\{\wedge, \square, \diamond$,false $\})$ satisfiability is coNP-complete. It follows from careful inspection of the proof of [3. Theorem 3.3] that satisfiability for variable-free $\mathcal{L}(\{\wedge, \square, \diamond$, true, false $\})$ formulas is coNP-hard.

Let $\phi$ be an $\mathcal{L}(\{\wedge, \square, \diamond$, true, false $\})$ formula. It is easy to see that $\phi$ is satisfiable if and only if the $\mathcal{L}(\{\wedge, \square, \diamond$, false $\})$ formula $\phi[$ true $:=t] \wedge \bigwedge_{i=0}^{m d(\phi)} \square^{i} t$ is satisfiable. 
Theorem 6.4 If $S \quad$ is $a$ subset of $\quad\{\neg,-, \square, \diamond$, true,false $\}, \quad\{-, \vee, \square, \diamond$, true, false $\}$, $\{-, \wedge, \square$, true,false $\}, \quad\{-, \wedge, \diamond$, true,false $\}, \quad\{\wedge, \vee, \square$, true,false $\}, \quad\{\wedge, \vee, \diamond$, true, false $\}, \quad$ or $\{\wedge, \vee, \square, \diamond$, true $\}$, then $\mathcal{L}(S)$ satisfiability is in $P$.

\section{Proof.}

1. Every $\mathcal{L}(\{\neg,-, \square, \diamond$, true, false $\})$ formula can in polynomial time be transformed into an equivalent $\mathcal{L}(\{-, \square, \diamond$, true,false $\})$ formula, by moving the negations inward. Since all operators are unary, every $\mathcal{L}(\{-, \square, \diamond$, true, false $\})$ formula is of the form $\{\square, \diamond\}^{*} a$, where $a$ is a literal or a constant. It is easy to see that the unsatisfiable formulas of this form are exactly the formulas of the form $\diamond^{*}$ false.

2. $\mathcal{L}(\{-, \vee, \square, \diamond$, true, false $\})$ formulas are of the form $\square \psi_{1} \vee \cdots \vee \square \psi_{k} \vee \diamond \xi_{1} \vee \cdots \vee \diamond \xi_{m} \vee a_{1} \vee \cdots \vee a_{s}$, where the $a_{i} \mathrm{~s}$ are literals or constants, and the $\psi_{i} \mathrm{~s}$ and $\xi_{i} \mathrm{~s}$ are $\mathcal{L}(\{-, \vee, \square, \diamond$, true, false $\})$ formulas.

If $k>0$ or if one of the $a_{i}$ s is a literal or true, this formula is satisfiable. Otherwise, the formula is satisfiable if and only if one of the $\xi_{i}$ s is satisfiable. This gives a recursive polynomial-time algorithm.

3. $\mathcal{L}(\{-, \wedge, \square$, true, false $\})$ formulas are of the form $\square \psi_{1} \wedge \cdots \wedge \square \psi_{k} \wedge a_{1} \wedge \cdots \wedge a_{s}$, where the $a_{i} \mathrm{~s}$ are literals or constants. This formula is satisfiable if and only if $a_{1} \wedge \cdots \wedge a_{s}$ is satisfiable.

4. $\mathcal{L}(\{-, \wedge, \diamond$, true, false $\})$ formulas are of the form: $\diamond \xi_{1} \wedge \cdots \wedge \diamond \xi_{m} \wedge a_{1} \wedge \cdots \wedge a_{s}$, where the $a_{i} \mathrm{~s}$ are literals or constants. This formula is satisfiable if and only if $a_{1} \wedge \cdots \wedge a_{s}$ is satisfiable and for every $i, \xi_{i}$ is satisfiable. This is gives a recursive polynomial-time algorithm.

5. An $\mathcal{L}(\{\wedge, \vee, \square$, true, false $\})$ formula is satisfiable if and only if substituting true for every propositional variable and for every outermost $\square$ subformula makes the formula true.

6. Satisfiability for $\mathcal{L}(\{\wedge, \vee, \diamond$, true, false $\})$ formulas can be recursively computed as follows. Replace every outermost $\diamond \psi$ subformula by true if $\psi$ is satisfiable and by false if $\psi$ is not satisfiable and replace all propositional variables by true. $\phi$ is satisfiable if and only if the resulting propositional sentence evaluates to true.

7. Every $\mathcal{L}(\{\wedge, \vee, \square, \diamond$, true $\})$ formula is satisfiable.

Note that it follows from Theorem 6.3(2) and Theorem 6.4(7) that adding the constant false can increase the complexity. It follows from the next theorem that the complexity can increase from $\mathrm{P}$ to PSPACE-complete.

Theorem 6.5 If $S \subseteq\{\neg,-, \wedge, \vee, \square, \diamond$, true, false $\}$ contains $\{\wedge, \vee, \square, \diamond$, false $\}$, then $\mathcal{L}(S)$ satisfiability is PSPACE-complete.

We will in fact prove the following theorem. 
Theorem 6.6 Satisfiability for modal formulas without literals (but with constants true and false) is PSPACE-complete.

This almost immediately implies Theorem 6.5. For let $\phi$ be a formula without propositional variables. Let $\phi^{\prime}$ be the formula that results from $\phi$ by bringing $\phi$ into a negation-less normal form. Then $\phi$ is satisfiable if and only if $\phi^{\prime}[$ true $:=t] \wedge \bigwedge_{i=0}^{m d(\phi)} \square^{i} t$ is satisfiable. This proves PSPACE-hardness. The corresponding upper bound again follows from Ladner [7].

Proof. We will use the following theorem.

Theorem 6.7 (Halpern [5]) Satisfiability for modal formulas with one propositional variable is PSPACE-complete.

We will reduce satisfiability for modal formulas with one propositional variable to satisfiability for modal formulas with zero propositional variables. The reduction has the same flavor as the proof of Theorem 6.7 from [5], in that we will encode the truth of the propositional variable by the frame.

Let $\phi$ be a modal formula with one propositional variable. We assume that $\phi$ is built from the sole propositional variable $p$, and operations $\{\neg, \wedge, \square\}$.

The main idea of the reduction is the following. Suppose $\phi$ is satisfiable. Then $\phi$ is satisfiable on a model $M=\langle W, R, \pi>$ in which every path has length $\leq \operatorname{md}(\phi)$. We extend $M$ in such a way that the assignment to $p$ is encoded in the frame. Define $M^{\prime}=<W^{\prime}, R^{\prime}, \pi^{\prime}>$ as follows.

- $W^{\prime}=W \cup\left\{w_{1}, w_{2}, \ldots, w_{m d(\phi)+1}\right\}$.

- $R^{\prime}=R \cup\left\{<w_{i}, w_{i+1}>\mid 1 \leq i \leq m d(\phi)\right\} \cup\left\{<w, w_{1}>\mid w \in W\right.$ and $\left.M, w \models p\right\}$.

- $\pi^{\prime}$ is irrelevant, since there are no propositional variables.

Thus, $M^{\prime}$ contains all the information of $M$, and there is a maximal path of length $m d(\phi)+1$ from a world $w \in W$ in $M^{\prime}$ if and only if $M, w \models p$. We will simulate $p$ by formula $\diamond^{m d(\phi)+1} \square$ false.

Suppose $M, w \models \square \psi$ for an arbitrary subformula $\square \psi$ of $\phi$. In the reduction, we need to make sure that we do not force $\psi$ to be true on the new world $w_{1}$. So, we will enforce that $\psi$ holds in all successor worlds that do not have a maximal path of length $m d(\phi)$. Let $f(\phi)=f_{m d(\phi)}(\phi)$, where $f_{k}(\phi)$ is defined inductively as follows.

- $f_{k}(p)=\diamond^{k+1} \square$ false

- $f_{k}(\neg \psi)=\neg f_{k}(\psi)$

- $f_{k}(\psi \wedge \xi)=f_{k}(\psi) \wedge f_{k}(\xi)$

- $f_{k}(\square \psi)=\square\left(\diamond^{k} \square\right.$ false $\left.\vee f_{k}(\psi)\right)$ 
It is straightforward to show that for all $w \in W$ and all formulas $\psi$ with $p$ as only propositional variable and such that $m d(\psi) \leq m d(\phi), M, w \models \psi$ if and only if $M^{\prime}, w \models f_{m d(\phi)}(\psi)$. This implies that if $\phi$ is satisfiable, then $f(\phi)$ is satisfiable.

For the converse, suppose that $f(\phi)$ is satisfiable. Let $M^{\prime}=\left\langle W^{\prime}, R^{\prime}, \pi^{\prime}\right\rangle$ be an acyclic model and $w_{0} \in W^{\prime}$ such that $M^{\prime}, w_{0} \models f(\phi)$. Define $M=<W, R, \pi>$ as follows.

- $W=\left(W^{\prime} \backslash\left\{w \in W^{\prime} \mid M^{\prime}, w \models \diamond^{m d(\phi)} \square\right.\right.$ false $\left.\}\right) \cup\left\{w_{0}\right\}$.

- $R=R^{\prime} \cap(W \times W)$.

- $\pi(p)=\left\{w \in W \mid M^{\prime}, w \models \diamond^{m d(\phi)+1} \square\right.$ false $\}$.

Again, it is easy to show that for all $w \in W$ and all formulas $\psi$ with $p$ as only propositional variable and such that $m d(\psi) \leq m d(\phi), M, w \models \psi$ if and only if $M^{\prime}, w \models f_{m d(\phi)}(\psi)$.

It follows that $\phi$ is satisfiable if and only if $f(\phi)$ is satisfiable. This proves the theorem, since $f$ is clearly computable in polynomial time.

It remains to show that we covered all cases.

Theorem 6.8 If $S \subseteq\left\{\neg,{ }^{-}, \wedge, \vee, \square, \diamond\right.$, true, false $\}$, then one of Theorems [6.1, 6.2, 6.3, 6.4, or 6.5 applies.

Proof. First suppose that $\neg \in S$. If Theorem 6.1 does not apply, then $S \subseteq\left\{\neg,{ }^{-}, \square, \diamond\right.$, true, false $\}$ or $S \subseteq\{\neg,-, \wedge, \vee$, true, false $\}$. In the first case, Theorem 6.4(1) applies. For the second case, either Theorem 6.2 applies or $S \subseteq\left\{\neg,{ }^{-}\right.$, true, false $\}$, in which case Theorem 6.4(1) applies.

Next suppose that $\neg \notin S$ and $-\in S$. If Theorem 6.4 does not apply, then $S$ is not a subset of $\{-, \vee, \square, \diamond$, true, false $\},\{-\wedge, \square$, true, false $\}$, or $\left\{{ }^{-}, \wedge, \diamond\right.$, true, false $\}$. This implies that $S$ contains $\{-, \wedge, \vee\}$ or $\{-, \wedge, \square, \diamond\}$. In the first case, Theorem 6.1 applies or Theorem 6.2(2) applies. In the second case, Theorem 6.1 applies or Theorem 6.3 (1) applies.

Finally, suppose that $\neg \notin S$ and ${ }^{-} \notin S$. That is, we are in a fragment of positive modal logic (see for example Dunn [4]). If Theorem 6.4 does not apply, then false $\in S$, since $S \nsubseteq\{\wedge, \vee, \square, \diamond$, true $\}$. Also, $\wedge \in S$, since $S \nsubseteq\{-, \vee, \square, \diamond$, true, false $\}$. If Theorem 6.4 does not apply, then $S \backslash\{\wedge$, false $\}$ is not a subset of $\{\vee, \square$, true $\}$ or of $\{\vee, \diamond$, true $\}$. It follows that $S$ contains $\{\wedge, \square, \diamond$, false $\}$ and Theorem 6.3 (2) or Theorem 6.5 applies.

\section{Acknowledgments}

I would like to thank Johan van Benthem for suggesting this topic, Michael Bauland, Johan van Benthem, Hans de Nivelle, Maarten de Rijke, Henning Schnoor, and Ilka Schnoor for helpful conversations and suggestions, and Stephan Tobies and the anonymous referees for useful comments and suggestions. 


\section{References}

[1] S. Demri and Ph. Schnoebelen. The complexity of propositional linear temporal logics in simple cases (extended abstract). In Proceedings of the 15th Annual Symposium on Theoretical Aspects of Computer Science, pp. 61-72, 1998.

[2] F. Donini, M. Lenzerini, D. Nardi, and W. Nutt. The complexity of concept languages. Information and Computation, 134, pp. 1-58, 1997.

[3] F. Donini, B. Hollunder, M. Lenzerini, D. Nardi, W. Nutt, and A. Spaccamela. The complexity of existential quantification in concept languages. Artificial Intelligence, 53(2-3), pp. 309-327, 1992.

[4] M. Dunn. Positive modal logic. Studia Logica, 55, pp. 301-317, 1995.

[5] J. Halpern. The effect of bounding the number of primitive propositions and the depth of nesting on the complexity of modal logic. Artificial Intelligence, 75(2), pp. 361-372, 1995.

[6] E. Hemaspaandra. The complexity of poor man's logic. Journal of Logic and Computation, 11(4), pp. 609-622, 2001.

[7] R. Ladner. The computational complexity of provability in systems of modal propositional logic. SIAM Journal on Computing, 6(3), pp. 467-480, 1977.

[8] K. Schild. A correspondence theory for terminological logics: preliminary report. In Proceedings of the 12th International Joint Conference on Artificial Intelligence, pp. 466-471, 1991.

[9] M. Schmidt-Schauss and G. Smolka. Attributive concept descriptions with complements. Artificial Intelligence, 48(1), pp. 1-26, 1991.

[10] L. Stockmeyer. The polynomial-time hierarchy. Theoretical Computer Science, 3, pp. 1-22, 1977.

[11] S. Tobies. PSPACE reasoning for graded modal logics. Journal of Logic and Computation, 11(1), pp. 85-106, 2001. 